\title{
Controlling Nanopore Size and Shape by \\ Fluorosurfactant templating of Silica
}

\section{Supporting Information}

Bing Tan ${ }^{\dagger}$, Hans-Joachim Lehmler ${ }^{\ddagger}$, Sandhya M. Vyas ${ }^{\ddagger}$, Barbara L. Knutson ${ }^{\dagger}$ and Stephen E. Rankin ${ }^{\dagger}$

${ }^{+}$Chemicals and Materials Engineering Department, University of

Kentucky, Lexington, KY, 40536-0046

$\ddagger$ Department of Occupational and Environmental Health, University of Iowa, Iowa City, IA, 52242-5000 


\section{Fluorocarbon Surfactant Synthesis}

The fluorocarbon surfactant was synthesized as described previously by alkylation of anhydrous pyridine with $1 H, 1 H, 2 H, 2 H$-perfluorooctyl iodide.[1] The resulting pyridinium iodide was carefully washed with anhydrous diethyl ether and recrystallized from acetone. The corresponding chloride was obtained by ion exchange chromatography on Amberlite IRA-410 and used without further purification. Melting points were determined using a Thermal Analysis 2920 Differential Scanning Instrument. Thermograms were recorded using a heating rate of $20^{\circ}$ minute from 20 to $230^{\circ} \mathrm{C}$ and the onset temperatures of the main transitions after one DSC run were determined.[2] Mass spectra were recorded at the University of Kentucky Mass Spectrometry Facility using a Kratos Kompact SEQ or Bruker Autoflex time-of-flight mass Spectrometer equipped with MALDI.

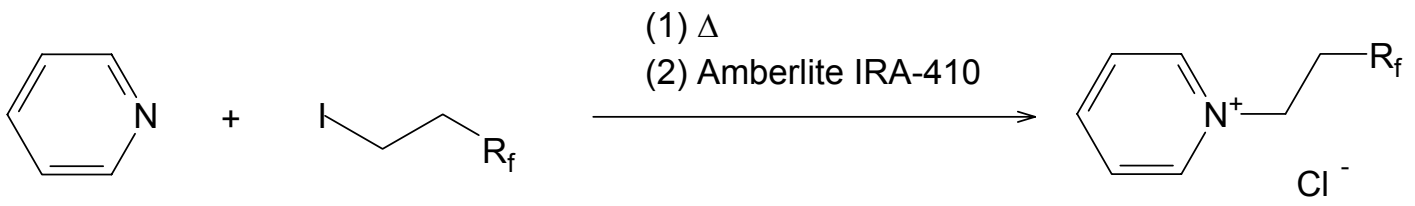

\section{References}

[1] T. Asakawa, H. Hisamatsu, S. Miyagishi, Micellar pseudophase separation regions of $1 H, 1 H, 2 H, 2 H$-perfluoroalkylpyridinium chloride and hydrocarbon surfactants by group contribution method, Langmuir 11 (1995) 478-482.

[2] H.-J. Lehmler, M.O. Oyewumi, M. Jay, P.M. Bummer, Behavior of partially fluorinated carboxylic acids at the air-water interface, J. Fluorine Chem. 107 (2001) 141-146. 
Table 1: Analytical data of the $1 H, 1 H, 2 H, 2 H$-perfluoroalkyl pyridinium iodides.

\begin{tabular}{|c|c|c|c|c|c|}
\hline $\begin{array}{c}1 \mathrm{H}, 1 \mathrm{H}, 2 \mathrm{H}, 2 \mathrm{H}- \\
\text { Perfluoroalkyl pyridinium } \\
\text { iodide }\end{array}$ & $\begin{array}{c}\text { Molecular } \\
\text { Formula }\end{array}$ & $\begin{array}{c}\text { Formula Weight } \\
{[\mathrm{g} / \mathrm{m}]}\end{array}$ & m.p. [ $\left.{ }^{\circ} \mathrm{C}\right]$ & $\begin{array}{c}\text { MS of } \\
\text { pyridinium ion } \\
\text { (calculated) }\end{array}$ & Combustion analysis \\
\hline $\mathrm{C}_{4} \mathrm{~F}_{9}\left(\mathrm{CH}_{2}\right)_{2-}$ & $\mathrm{C}_{11} \mathrm{~F}_{9} \mathrm{H}_{9} \mathrm{NI}$ & 452.97 & $122^{\#}$ & $326(326)$ & $\begin{array}{c}\text { Calcd.: C 29.14, H 2.00, N 3.09 } \\
\text { Found: C 29.12, H 1.93, N 3.33 }\end{array}$ \\
\hline $\mathrm{C}_{6} \mathrm{~F}_{13}\left(\mathrm{CH}_{2}\right)_{2^{-}}$ & $\mathrm{C}_{13} \mathrm{~F}_{13} \mathrm{H}_{9} \mathrm{NI}$ & 552.96 & $\begin{array}{c}110,130,170 \\
\text { and 186 }\end{array}$ & $426(426)$ & {$[1]$} \\
\hline $\mathrm{C}_{8} \mathrm{~F}_{17}\left(\mathrm{CH}_{2}\right)_{2^{-}}$ & $\mathrm{C}_{15} \mathrm{~F}_{17} \mathrm{H}_{9} \mathrm{NI}$ & 652.96 & 150,197 & $526(526)$ & {$[1]$} \\
\hline $\mathrm{C}_{10} \mathrm{~F}_{21}\left(\mathrm{CH}_{2}\right)_{2-}$ & $\mathrm{C}_{17} \mathrm{~F}_{21} \mathrm{H}_{9} \mathrm{NI}$ & 752.95 & 172,208 & $626(626)$ & $\begin{array}{c}\text { Calcd.: C 27.09, H 1.20, N 1.86 } \\
\text { Found: C 27.12, H 1.14, N 1.88 }\end{array}$ \\
\hline$\left(\mathrm{F}_{3} \mathrm{C}\right)_{2} \mathrm{CF}_{\left(\mathrm{CF}_{2}\right)_{4}\left(\mathrm{CH}_{2}\right)_{2-}-}$ & $\mathrm{C}_{14} \mathrm{~F}_{15} \mathrm{H}_{9} \mathrm{NI}$ & 602.96 & $173^{*}, 191$ & $476(476)$ & $\begin{array}{c}\text { Calcd.: } \mathrm{C} \mathrm{27.86,} \mathrm{H} \mathrm{1.50,} \mathrm{N} \mathrm{2.32} \\
\text { Found: C 27.87, H 1.45, N 2.38 }\end{array}$ \\
\hline$\left(\mathrm{F}_{3} \mathrm{C}\right)_{2} \mathrm{CF}\left(\mathrm{CF}_{2}\right)_{6}\left(\mathrm{CH}_{2}\right)_{2-}-$ & $\mathrm{C}_{16} \mathrm{~F}_{19} \mathrm{H}_{9} \mathrm{NI}$ & 702.95 & $124^{*}, 186$ & $576(576)$ & $\begin{array}{c}\text { Calcd.: C 27.31, H 1.29, N 1.99 } \\
\text { Found: C 27.49, H 1.21, N 2.06 }\end{array}$ \\
\hline
\end{tabular}

* Minor phase transition.

\# Two broad peaks with maxima at 125 and $141^{\circ} \mathrm{C}$. 
Table 2: ${ }^{1} \mathrm{H},{ }^{13} \mathrm{C}$ and ${ }^{19} \mathrm{~F}$ NMR of the $1 \mathrm{H}, 1 \mathrm{H}, 2 \mathrm{H}, 2 \mathrm{H}$-perfluoroalkyl pyridinium iodides in $\mathrm{d}_{6}$-acetone at 400,100 and $282 \mathrm{MHz}$, respectively.

\begin{tabular}{|c|c|c|c|}
\hline $\begin{array}{c}1 \mathrm{H}, 1 \mathrm{H}, 2 \mathrm{H}, 2 \mathrm{H}- \\
\text { Perfluoroalkyl } \\
\text { pyridinium iodide }\end{array}$ & ${ }^{1} \mathrm{H}$ NMR $[\mathrm{ppm}]$ & ${ }^{13} \mathrm{C}$ NMR [ppm] & ${ }^{19} \mathrm{~F} \mathrm{NMR}^{\#}[\mathrm{ppm}]$ \\
\hline $\mathrm{C}_{4} \mathrm{~F}_{9}\left(\mathrm{CH}_{2}\right)_{2-}$ & $\begin{array}{c}3.1-3.3\left(\mathrm{~m},-\mathrm{CH}_{2} \mathrm{CF}_{2-}, 2 \mathrm{H}\right), 5.12\left(\mathrm{t},-\mathrm{CH}_{2} \mathrm{CH}_{2} \mathrm{CF}_{2},\right. \\
J=7.3 \mathrm{~Hz}), 8.23(\text { " t", } J \sim 7.2 \mathrm{~Hz}, 2 \mathrm{H}), 8.71(\mathrm{t}, J= \\
7.8 \mathrm{~Hz}, \mathrm{t}, J=1.2 \mathrm{~Hz}, 1 \mathrm{H}), 9.21(\text { ("d", } J \sim 5.6 \mathrm{~Hz}, \\
2 \mathrm{H})\end{array}$ & $\begin{array}{c}32.75\left(\mathrm{t},-\mathrm{CH}_{2} \mathrm{CF}_{2-}, J_{\mathrm{CF}}=21.1 \mathrm{~Hz}\right) \\
55.17\left(\mathrm{t},-\mathrm{CH}_{2} \mathrm{CH}_{2} \mathrm{CF}_{2}\right), 129.93 \\
146.84,148.05\end{array}$ & $\begin{array}{c}-80.8\left(-\mathrm{CF}_{3}\right) \\
-113.1,-123.9 \\
-125.7\end{array}$ \\
\hline $\mathrm{C}_{6} \mathrm{~F}_{13}\left(\mathrm{CH}_{2}\right)_{2-}$ & $\begin{array}{c}3.1-3.3\left(\mathrm{~m},-\mathrm{CH}_{2} \mathrm{CF}_{2-}, 2 \mathrm{H}\right), 5.01\left(\mathrm{t},-\mathrm{CH}_{2} \mathrm{CH}_{2} \mathrm{CF}_{2},\right. \\
J=7.2 \mathrm{~Hz}), 8.23(\text { 't", } J \sim 7.0 \mathrm{~Hz}, 2 \mathrm{H}), 8.68(\mathrm{t}, J= \\
8.0 \mathrm{~Hz}, \mathrm{t}, J=1.2 \mathrm{~Hz}, 1 \mathrm{H}), 9.23(\text { (“d”, } J \sim 5.2 \mathrm{~Hz}, \\
2 \mathrm{H})\end{array}$ & $\begin{array}{c}32.70\left(\mathrm{t},-\mathrm{CH}_{2} \mathrm{CF}_{2^{-}}, J_{\mathrm{CF}}=20 \mathrm{~Hz}\right) \\
55.10\left(\mathrm{t},-\mathrm{CH}_{2} \mathrm{CH}_{2} \mathrm{CF}_{2}\right), 129.89 \\
146.82,148.01\end{array}$ & $\begin{array}{c}-80.6\left(-\mathrm{CF}_{3}\right) \\
-112.6,-121.3 \\
-122.3,-122.7,- \\
125.7\end{array}$ \\
\hline $\mathrm{C}_{8} \mathrm{~F}_{17}\left(\mathrm{CH}_{2}\right)_{2-}$ & $\begin{array}{c}3.1-3.3\left(\mathrm{~m},-\mathrm{CH}_{2} \mathrm{CF}_{2}-, 2 \mathrm{H}\right), 5.02\left(\mathrm{t},-\mathrm{CH}_{2} \mathrm{CH}_{2} \mathrm{CF}_{2},\right. \\
J=7.2 \mathrm{~Hz}), 8.23(\text { " t", } J \sim 7.0 \mathrm{~Hz}, 2 \mathrm{H}), 8.68(\mathrm{t}, J= \\
8.0 \mathrm{~Hz}, \mathrm{t}, J=1.2 \mathrm{~Hz}, 1 \mathrm{H}), 9.23(\text { ("d", } J \sim 5.2 \mathrm{~Hz}, \\
2 \mathrm{H})\end{array}$ & $\begin{array}{c}32.72\left(\mathrm{t},-\mathrm{CH}_{2} \mathrm{CF}_{2-}, J_{\mathrm{CF}}=20 \mathrm{~Hz}\right) \\
55.11\left(\mathrm{t},-\mathrm{CH}_{2} \mathrm{CH}_{2} \mathrm{CF}_{2}\right), 129.89 \\
146.81,148.02\end{array}$ & $\begin{array}{c}-81.5\left(-\mathrm{CF}_{3}\right) \\
-113.2,-122.2(3 \times \\
\left.\mathrm{CF}_{2}\right),-122.6,- \\
123.4,-126.4\end{array}$ \\
\hline $\mathrm{C}_{10} \mathrm{~F}_{21}\left(\mathrm{CH}_{2}\right)_{2^{-}}$ & $\begin{array}{c}3.1-3.3\left(\mathrm{~m},-\mathrm{CH}_{2} \mathrm{CF}_{2^{-}}, 2 \mathrm{H}\right), 5.08\left(\mathrm{t},-\mathrm{CH}_{2} \mathrm{CH}_{2} \mathrm{CF}_{2},\right. \\
J=7.2 \mathrm{~Hz}), 8.19 \text { ("t"', } J \sim 7.2 \mathrm{~Hz}, 2 \mathrm{H}), 8.68(\mathrm{t}, J= \\
7.8 \mathrm{~Hz}, \mathrm{t}, J \sim 1.2 \mathrm{~Hz}, 1 \mathrm{H}), 9.153 \text { ("d"), } J \sim 5.9 \mathrm{~Hz}, \\
2 \mathrm{H})\end{array}$ & $\begin{array}{c}32.97\left(\mathrm{t},-\mathrm{CH}_{2} \mathrm{CF}_{2-}, J_{\mathrm{CF}}=20 \mathrm{~Hz}\right) \\
55.16\left(\mathrm{t},-\mathrm{CH}_{2} \mathrm{CH}_{2} \mathrm{CF}_{2}\right), 129.91 \\
146.82,148.05\end{array}$ & $\begin{array}{c}-80.5\left(-\mathrm{CF}_{3}\right) \\
-112.6,-120.9(5 \times \\
\left.\mathrm{CF}_{2}\right),-121.9 \\
-122.5,-125.4\end{array}$ \\
\hline$\left(\mathrm{F}_{3} \mathrm{C}\right)_{2} \mathrm{CF}\left(\mathrm{CF}_{2}\right)_{4}\left(\mathrm{CH}_{2}\right)_{2}-$ & $\begin{array}{c}3.1-3.3\left(\mathrm{~m},-\mathrm{CH}_{2} \mathrm{CF}_{2-}, 2 \mathrm{H}\right), 5.07\left(\mathrm{t},-\mathrm{CH}_{2} \mathrm{CH}_{2} \mathrm{CF}_{2},\right. \\
J=7.2 \mathrm{~Hz}), 8.17 \text { (“t”, } J \sim 7.0 \mathrm{~Hz}, 2 \mathrm{H}), 8.66(\mathrm{t}, J= \\
7.8 \mathrm{~Hz}, \mathrm{t}, J \sim 1.2 \mathrm{~Hz}, 1 \mathrm{H}), 9.15 \text { (“d”, } J \sim 5.2 \mathrm{~Hz}, \\
2 \mathrm{H})\end{array}$ & $\begin{array}{c}32.85\left(\mathrm{t},-\mathrm{CH}_{2} \mathrm{CF}_{2-}, J_{\mathrm{CF}}=20 \mathrm{~Hz}\right) \\
55.21\left(\mathrm{t},-\mathrm{CH}_{2} \mathrm{CH}_{2} \mathrm{CF}_{2}\right), 129.94 \\
146.83,148.04\end{array}$ & $\begin{array}{l}-71.4\left(2 \times \mathrm{CF}_{3}\right) \\
-112.6,-114.1 \\
-119.7,-122.0 \\
-185.3(\mathrm{CF})\end{array}$ \\
\hline$\left(\mathrm{F}_{3} \mathrm{C}\right)_{2} \mathrm{CF}\left(\mathrm{CF}_{2}\right)_{6}\left(\mathrm{CH}_{2}\right)_{2-}$ & $\begin{array}{c}3.1-3.3\left(\mathrm{~m},-\mathrm{CH}_{2} \mathrm{CF}_{2^{-}}, 2 \mathrm{H}\right), 5.12\left(\mathrm{t},-\mathrm{CH}_{2} \mathrm{CH}_{2} \mathrm{CF}_{2},\right. \\
J=7.3 \mathrm{~Hz}), 8.21(\text { 't", } J \sim 7.2 \mathrm{~Hz}, 2 \mathrm{H}), 8.70(\mathrm{t}, J= \\
7.8 \mathrm{~Hz}, \mathrm{t}, J=1.2 \mathrm{~Hz}, 1 \mathrm{H}), 9.20 \text { (“d”, } J \sim 5.6 \mathrm{~Hz}, \\
2 \mathrm{H})\end{array}$ & $\begin{array}{c}32.87\left(\mathrm{t},-\mathrm{CH}_{2} \mathrm{CF}_{2^{-}}, J_{\mathrm{CF}}=21.2 \mathrm{~Hz}\right) \\
55.22\left(\mathrm{t},-\mathrm{CH}_{2} \mathrm{CH}_{2} \mathrm{CF}_{2}\right), 129.95 \\
146.86,148.05\end{array}$ & $\begin{array}{c}-71.7\left(2 \times \mathrm{CF}_{3}\right), \\
-112.5,-114.2 \\
-119.8,-120.7(2 \times \\
\left.\mathrm{CF}_{3}\right),-122.7 \\
-185.7(\mathrm{CF})\end{array}$ \\
\hline
\end{tabular}


\# Chemical shifts were determined using $\mathrm{CFCl}_{3}$ as internal standard. 
Table 3: An analytical data of the $1 H, 1 H, 2 H, 2 H$-perfluoroalkyl pyridinium chlorides.

\begin{tabular}{|c|c|c|c|c|c|}
\hline $\begin{array}{c}1 H, 1 H, 2 H, 2 H- \\
\text { Perfluoroalkyl pyridinium } \\
\text { chloride } \\
\text { (sample code) }\end{array}$ & $\begin{array}{l}\text { Molecular } \\
\text { Formula }\end{array}$ & $\begin{array}{c}\text { Formula } \\
\text { Weight } \\
{[\mathrm{g} / \mathrm{m}]}\end{array}$ & m.p. $\left[{ }^{\circ} \mathrm{C}\right]$ & MS (calculated) & Combustion analysis \\
\hline $\begin{array}{c}\mathrm{C}_{4} \mathrm{~F}_{9}\left(\mathrm{CH}_{2}\right)_{2-} \\
(\mathrm{UK} 6)\end{array}$ & $\mathrm{C}_{11} \mathrm{~F}_{9} \mathrm{H}_{9} \mathrm{NCl}$ & 361.52 & 160 & $326(326)$ & $\begin{array}{l}\text { Calcd.: C } 36.51, \text { H } 2.51, \text { N } 3.87 \\
\text { Found: C } 36.13, \text { H } 2.50, \text { N } 3.94\end{array}$ \\
\hline $\begin{array}{c}\mathrm{C}_{6} \mathrm{~F}_{13}\left(\mathrm{CH}_{2}\right)_{2-} \\
(\mathrm{UK} 8)\end{array}$ & $\mathrm{C}_{13} \mathrm{~F}_{13} \mathrm{H}_{9} \mathrm{NCl}$ & 461.51 & 178 & $426(426)$ & ( \\
\hline $\begin{array}{l}\mathrm{C}_{8} \mathrm{~F}_{17}\left(\mathrm{CH}_{2}\right)_{2-}^{-} \\
(\mathrm{UK} 10)\end{array}$ & $\mathrm{C}_{15} \mathrm{~F}_{17} \mathrm{H}_{9} \mathrm{NCl}$ & 561.50 & 165,172 & $526(526)$ & {$[1]$} \\
\hline $\begin{array}{c}\mathrm{C}_{10} \mathrm{~F}_{21}\left(\mathrm{CH}_{2}\right)_{2^{-}} \\
(\mathrm{UK} 12)\end{array}$ & $\begin{array}{c}\mathrm{C}_{17} \mathrm{~F}_{21} \mathrm{H}_{9} \mathrm{NCl} \cdot \\
\mathrm{H}_{2} \mathrm{O}\end{array}$ & 661.50 & $81^{*}, 126^{\#}, 198$ & $626(626)$ & $\begin{array}{l}\text { Calcd.: C 30.02, H 1.61, N } 2.06 \\
\text { Found: C 29.87, H 1.56, N } 2.05\end{array}$ \\
\hline $\begin{array}{c}\left(\mathrm{F}_{3} \mathrm{C}\right)_{2} \mathrm{CF}\left(\mathrm{CF}_{2}\right)_{4}\left(\mathrm{CH}_{2}\right)_{2^{-}} \\
(\mathrm{UK} 9)\end{array}$ & $\begin{array}{c}\mathrm{C}_{14} \mathrm{~F}_{15} \mathrm{H}_{9} \mathrm{NCl} \cdot \\
\mathrm{H}_{2} \mathrm{O}\end{array}$ & 511.51 & 106 & $476(476)$ & $\begin{array}{l}\text { Calcd.: C 31.72, H 2.07, N } 2.64 \\
\text { Found: C 31.70, H 1.97, N } 2.55\end{array}$ \\
\hline $\begin{array}{c}\left(\mathrm{F}_{3} \mathrm{C}\right)_{2} \mathrm{CF}\left(\mathrm{CF}_{2}\right)_{6}\left(\mathrm{CH}_{2}\right)_{2-} \\
(\mathrm{UK} 11)\end{array}$ & $\mathrm{C}_{16} \mathrm{~F}_{19} \mathrm{H}_{9} \mathrm{NCl}$ & 611.50 & 105 & $576(576)$ & $\begin{array}{l}\text { Calcd.: C } 31.40, \text { H } 1.48, \text { N } 2.29 \\
\text { Found: C } 31.14, \text { H 1.46, N } 2.30 \\
\end{array}$ \\
\hline
\end{tabular}

* Minor phase transition.

${ }^{\#}$ Broad minor phase transition with maximum at $129^{\circ} \mathrm{C}$ and shoulder at $134^{\circ} \mathrm{C}$. 
Table 4: ${ }^{1} \mathrm{H},{ }^{13} \mathrm{C}$ and ${ }^{19} \mathrm{~F}$ NMR of the $1 H, 1 H, 2 H, 2 H$-perfluoroalkyl pyridinium chlorides in $\mathrm{d}_{6}$-acetone at 400,100 and 282 MHz, respectively.

\begin{tabular}{|c|c|c|c|}
\hline $\begin{array}{c}1 H, 1 H, 2 H, 2 H- \\
\text { Perfluoroalkyl pyridinium } \\
\text { chloride } \\
\text { (sample code) }\end{array}$ & ${ }^{1} \mathrm{H}$ NMR $[\mathrm{ppm}]$ & ${ }^{13} \mathrm{C}$ NMR $[\mathrm{ppm}]$ & ${ }^{19} \mathrm{~F} \mathrm{NMR}^{\#}[\mathrm{ppm}]$ \\
\hline $\begin{array}{l}\mathrm{C}_{4} \mathrm{~F}_{9}\left(\mathrm{CH}_{2}\right)_{2-} \\
(\mathrm{UK} 6)\end{array}$ & $\begin{array}{c}3.1-3.3\left(\mathrm{~m},-\mathrm{CH}_{2} \mathrm{CF}_{2^{-}}, 2 \mathrm{H}\right), 5.08(\mathrm{t},- \\
\left.\mathrm{CH}_{2} \mathrm{CH}_{2} \mathrm{CF}_{2}, J=7.2 \mathrm{~Hz}\right), 8.18(“ \mathrm{t} ", J \sim 7.2 \\
\mathrm{Hz}, 2 \mathrm{H}), 8.68(\mathrm{t}, J=7.8 \mathrm{~Hz}, \mathrm{t}, J=1.4 \mathrm{~Hz}, \\
1 \mathrm{H}), 9.18 \text { ("d", } J \sim 5.4 \mathrm{~Hz}, 2 \mathrm{H})\end{array}$ & $\begin{array}{c}32.51\left(\mathrm{t},-\mathrm{CH}_{2} \mathrm{CF}_{2^{-}}, J_{\mathrm{CF}}=21 \mathrm{~Hz}\right) \\
54.96\left(\mathrm{t},-\mathrm{CH}_{2} \mathrm{CH}_{2} \mathrm{CF}_{2}\right), 129.75 \\
146.67,147.87\end{array}$ & $\begin{array}{l}-80.8\left(-\mathrm{CF}_{3}\right) \\
-112.8,-123.5 \\
-125.4\end{array}$ \\
\hline $\begin{array}{c}\mathrm{C}_{6} \mathrm{~F}_{13}\left(\mathrm{CH}_{2}\right)_{2-} \\
(\mathrm{UK} 8)\end{array}$ & $\begin{array}{c}3.1-3.3\left(\mathrm{~m},-\mathrm{CH}_{2} \mathrm{CF}_{2^{-}}, 2 \mathrm{H}\right), 5.02(\mathrm{t},- \\
\left.\mathrm{CH}_{2} \mathrm{CH}_{2} \mathrm{CF}_{2}, J=7.2 \mathrm{~Hz}\right), 8.20 \text { ("'t", } J \sim 7.0 \\
\mathrm{~Hz}, 2 \mathrm{H}), 8.69(\mathrm{t}, J=8.0 \mathrm{~Hz}, \mathrm{t}, J=1.2 \mathrm{~Hz}, \\
1 \mathrm{H}), 9.18 \text { ("cd", } J \sim 5.2 \mathrm{~Hz}, 2 \mathrm{H})\end{array}$ & $\begin{array}{c}30.55\left(\mathrm{t},-\mathrm{CH}_{2} \mathrm{CF}_{2^{-}}, J_{\mathrm{CF}}=20 \mathrm{~Hz}\right) \\
52.83\left(\mathrm{t},-\mathrm{CH}_{2} \mathrm{CH}_{2} \mathrm{CF}_{2}\right), 128.07 \\
145.52,146.34\end{array}$ & $\begin{array}{l}-80.6\left(-\mathrm{CF}_{3}\right) \\
-112.6,-121.3 \\
-122.3,-122.8 \\
-125.7 \\
\end{array}$ \\
\hline $\begin{array}{l}\mathrm{C}_{8} \mathrm{~F}_{17}\left(\mathrm{CH}_{2}\right)_{2-} \\
\quad(\mathrm{UK} 10)\end{array}$ & $\begin{array}{c}3.1-3.3\left(\mathrm{~m},-\mathrm{CH}_{2} \mathrm{CF}_{2^{-}}, 2 \mathrm{H}\right), 5.07(\mathrm{t},- \\
\left.\mathrm{CH}_{2} \mathrm{CH}_{2} \mathrm{CF}_{2}, J=7.2 \mathrm{~Hz}\right), 8.18(\text { '“t”, } J \sim 7.2 \\
\mathrm{Hz}, 2 \mathrm{H}), 8.68(\mathrm{t}, J=7.8 \mathrm{~Hz}, \mathrm{t}, J=1.3 \mathrm{~Hz}, \\
1 \mathrm{H}), 9.18 \text { (“"d", } J \sim 5.5 \mathrm{~Hz}, 2 \mathrm{H})\end{array}$ & $\begin{array}{c}32.72\left(\mathrm{t},-\mathrm{CH}_{2} \mathrm{CF}_{2-}, J_{\mathrm{CF}}=21 \mathrm{~Hz}\right), \\
55.11\left(\mathrm{t},-\mathrm{CH}_{2} \mathrm{CH}_{2} \mathrm{CF}_{2}\right), 129.89, \\
146.81,148.02\end{array}$ & $\begin{array}{c}-81.1\left(-\mathrm{CF}_{3}\right) \\
-112.9,-122.6\left(3 \times \mathrm{CF}_{3}\right) \\
-123.1,-123.5,-126.5\end{array}$ \\
\hline $\begin{array}{l}\mathrm{C}_{10} \mathrm{~F}_{21}\left(\mathrm{CH}_{2}\right)_{2-} \\
\quad(\mathrm{UK} 12)\end{array}$ & $\begin{array}{c}3.1-3.3\left(\mathrm{~m},-\mathrm{CH}_{2} \mathrm{CF}_{2^{-}}, 2 \mathrm{H}\right), 5.10(\mathrm{t},- \\
\left.\mathrm{CH}_{2} \mathrm{CH}_{2} \mathrm{CF}_{2}, J=7.2 \mathrm{~Hz}\right), 8.20(“ \mathrm{t} ", J \sim 7.2 \\
\mathrm{Hz}, 2 \mathrm{H}), 8.69(\mathrm{t}, J=7.8 \mathrm{~Hz}, \mathrm{t}, J=1.3 \mathrm{~Hz}, \\
1 \mathrm{H}), 9.14 \text { ("d", } J \sim 5.6 \mathrm{~Hz}, 2 \mathrm{H})\end{array}$ & $\begin{array}{c}32.64\left(\mathrm{t},-\mathrm{CH}_{2} \mathrm{CF}_{2-}, J_{\mathrm{CF}}=21 \mathrm{~Hz}\right), \\
55.00\left(\mathrm{t},-\mathrm{CH}_{2} \mathrm{CH}_{2} \mathrm{CF}_{2}\right), 129.78, \\
146.71,147.89\end{array}$ & $\begin{array}{c}-80.5\left(-\mathrm{CF}_{3}\right) \\
-112.6,-120.9\left(5 \times \mathrm{CF}_{3}\right) \\
-121.9,-122.5,-125.4\end{array}$ \\
\hline $\begin{array}{c}\left(\mathrm{F}_{3} \mathrm{C}\right)_{2} \mathrm{CF}\left(\mathrm{CF}_{2}\right)_{4}\left(\mathrm{CH}_{2}\right)_{2}- \\
(\mathrm{UK} 9)\end{array}$ & $\begin{array}{c}3.1-3.3\left(\mathrm{~m},-\mathrm{CH}_{2} \mathrm{CF}_{2^{-}}, 2 \mathrm{H}\right), 5.07(\mathrm{t},- \\
\left.\mathrm{CH}_{2} \mathrm{CH}_{2} \mathrm{CF}_{2}, J=7.2 \mathrm{~Hz}\right), 8.19 \text { ("'t", } J \sim 7.2 \\
\mathrm{~Hz}, 2 \mathrm{H}), 8.69(\mathrm{t}, J=7.8 \mathrm{~Hz}, \mathrm{t}, J=1.3 \mathrm{~Hz}, \\
1 \mathrm{H}), 9.14 \text { (“d”, } J \sim 5.6 \mathrm{~Hz}, 2 \mathrm{H})\end{array}$ & $\begin{array}{c}32.96\left(\mathrm{t},-\mathrm{CH}_{2} \mathrm{CF}_{2^{-}}, J_{\mathrm{CF}}=21 \mathrm{~Hz}\right), \\
55.13\left(\mathrm{t},-\mathrm{CH}_{2} \mathrm{CH}_{2} \mathrm{CF}_{2}\right), 129.89, \\
146.80,148.02\end{array}$ & $\begin{array}{l}-71.7\left(2 \times \mathrm{CF}_{3}\right) \\
-112.5,-114.4 \\
-119.7,-122.2 \\
-185.3(\mathrm{CF})\end{array}$ \\
\hline $\begin{array}{c}\left(\mathrm{F}_{3} \mathrm{C}\right)_{2} \mathrm{CF}\left(\mathrm{CF}_{2}\right)_{6}\left(\mathrm{CH}_{2}\right)_{2}- \\
(\mathrm{UK} 11)\end{array}$ & $\begin{array}{c}3.1-3.3\left(\mathrm{~m},-\mathrm{CH}_{2} \mathrm{CF}_{2-}, 2 \mathrm{H}\right), 5.12(\mathrm{t},- \\
\left.\mathrm{CH}_{2} \mathrm{CH}_{2} \mathrm{CF}_{2}, J=7.2 \mathrm{~Hz}\right), 8.19 \text { (“"t", } J \sim 7.2 \\
\mathrm{~Hz}, 2 \mathrm{H}), 8.69(\mathrm{t}, J=7.8 \mathrm{~Hz}, \mathrm{t}, J=1.3 \mathrm{~Hz}, \\
1 \mathrm{H}), 9.24 \text { ("d"d", } J \sim 5.6 \mathrm{~Hz}, 2 \mathrm{H})\end{array}$ & $\begin{array}{c}32.75\left(\mathrm{t},-\mathrm{CH}_{2} \mathrm{CF}_{2^{-}}, J_{\mathrm{CF}}=21 \mathrm{~Hz}\right), \\
55.14\left(\mathrm{t},-\mathrm{CH}_{2} \mathrm{CH}_{2} \mathrm{CF}_{2}\right), 129.92, \\
146.82,148.05\end{array}$ & $\begin{array}{c}-71.5\left(2 \times \mathrm{CF}_{3}\right), \\
-112.7,-114.4 \\
-120.1,-121.0\left(2 \times \mathrm{CF}_{3}\right), \\
-122.8,-185.5 \quad(\mathrm{CF}) \\
\end{array}$ \\
\hline
\end{tabular}

\footnotetext{
${ }^{\#}$ Chemical shifts were determined using $\mathrm{CFCl}_{3}$ as internal standard.
} 